\title{
$\beta$-Catenin and E-Cadherin Expression Patterns in High-Grade Endometrial Carcinoma Are Associated with Histological Subtype
}

\author{
Peter W. Schlosshauer, M.D., Lora Hedrick Ellenson, M.D., Robert A. Soslow, M.D. \\ Department of Pathology, Weill Medical College of Cornell University, New York, New York
}

Both $\beta$-catenin and E-cadherin are epithelial cell adhesion molecules. In addition, $\beta$-catenin is an important element of the Wnt signal transduction pathway, which has been implicated in embryogenesis and carcinogenesis, including the development of endometrial and ovarian endometrioid carcinomas. We hypothesized that the expression pattern of these two adhesion molecules may depend upon the histological subtype of endometrial carcinomas. Therefore, we compared the immunohistochemical expression of $\beta$-catenin and E-cadherin in a set of uterine adenocarcinomas matched for high histologic grade, that is, poorly differentiated (International Federation of Gynecology and Obstetrics [FIGO] Grade III) uterine endometrioid carcinomas and uterine serous carcinomas. Seventeen FIGO Grade III endometrioid adenocarcinomas and 17 serous carcinomas were evaluated histologically and immunohistochemically with commercially available monoclonal antibodies against $\beta$-catenin and E-cadherin. Nuclear expression of $\beta$-catenin was observed in 8 of 17 (47\%) endometrioid adenocarcinomas but in none of the serous carcinomas $(P$ $=.003)$. Moderate or strong E-cadherin expression was identified in 7 of $17(41 \%)$ serous carcinomas as opposed to in only 1 of $17(6 \%)$ endometrioid adenocarcinomas $(P=.02)$. The majority of endometrioid adenocarcinomas showed strong $\beta$-catenin expression coupled with weak E-cadherin expression; serous carcinomas did not exhibit a comparable trend. Our results indicate that the expression of $\beta$-catenin and E-cadherin in high-grade endometrial cancers is strongly associated with histological subtype. These data provide further support for the

\footnotetext{
Copyright (C) 2002 by The United States and Canadian Academy of Pathology, Inc.

VOL. 15, NO. 10, P. 1032, 2002 Printed in the U.S.A.

Date of acceptance: June 21, 2002.

Peter W. Schlosshauer, M.D., is now at the Department of Pathology at the Mount Sinai School of Medicine, New York, New York.

Address reprint requests to: Robert A. Soslow, M.D., Department of Pa-

thology, Memorial Sloan Kettering Cancer Center, 1275 York Avenue, New

York, NY 10021; e-mail: soslowr@mskcc.org.

DOI: 10.1097/01.MP.0000028573.34289.04
}

distinct molecular profiles of endometrioid adenocarcinoma and serous carcinoma. Notably, differences in cell adhesion molecule expression could account for variations in patterns of tumor dissemination. The immunohistochemical staining pattern may also be useful for diagnostic purposes.

KEY WORDS: $\beta$-catenin, E-cadherin, Endometrial carcinoma, Histological subtypes.

Mod Pathol 2002;15(10):1032-1037

Based on clinical evidence, it has been suggested that carcinomas of the endometrium can be subdivided into estrogen-related (Type I) and nonestrogen-related (Type II) tumors (1, 2, 3). Morphologically, these two types are most frequently represented by the endometrioid and serous variants of endometrial carcinoma, respectively. More recently, molecular genetic evidence (e.g., the distribution of PTEN, K-ras, and p53 mutations and microsatellite instability) has accumulated, suggesting that different pathobiologic pathways are involved in the evolution of these two types $(4,5)$.

E-cadherin is a calcium-dependent transmembranous epithelial adhesion molecule, which is linked to cytoskeletal actin filaments through $\alpha$ and $\beta$ - (or alternatively $\gamma$-) catenin. $\beta$-catenin, in addition to its function in this cell adhesion complex, serves as a key element in the Wnt signal transduction pathway, which has been implicated in embryogenesis and carcinogenesis (reviewed in $6-11$ ). Cytosolic accumulation of $\beta$-catenin leads to its complex formation with transcription factors like Tcf/Lef-1, translocation into the nucleus, and induction of transcription of responsive genes, including $c$-myc (12), cyclin D1 (13), and $c$-jun and fra-1 (14; Fig. 1). Alterations in E-cadherin expression have been linked to decreased cell-cell adhesion, metastatic potential, tumor dedifferentiation, and deep myometrial invasion in endometrial and other carcinomas $(15,16)$. Similarly, mutations in the $\beta$-catenin-encoding gene CTNNB1 may lead to defective cell adhesion function (17). Previous stud- 


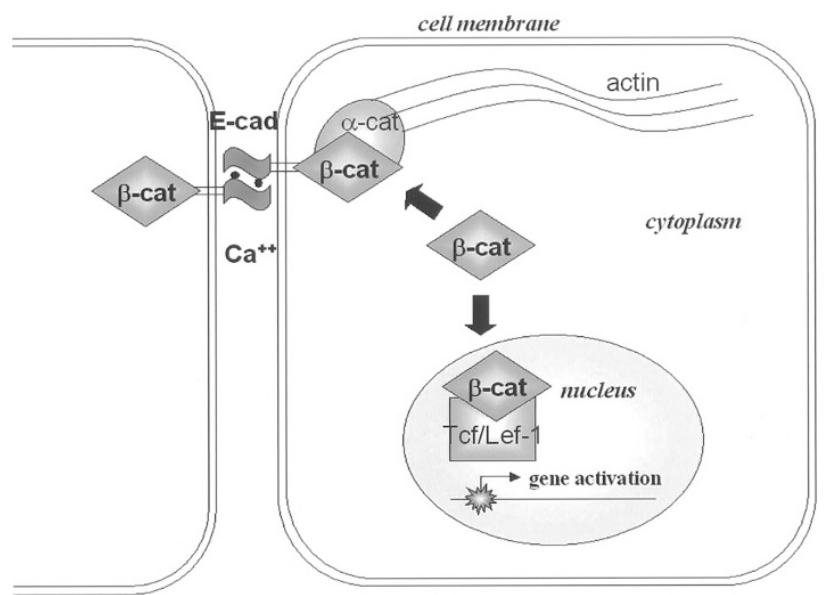

FIGURE 1. Subcellular localization and interactions of $\beta$-catenin, Ecadherin, and other molecules. Note that $\beta$-catenin functions both as a cell adhesion molecule and as a transcription cofactor.

ies have reported $\beta$-catenin mutations that lead to stabilization and accumulation of the molecule in $10-30 \%$ and more of both uterine and ovarian endometrioid adenocarcinomas (18-24). Alterations in $\beta$-catenin have not been reported in serous carcinomas.

To evaluate differences in the expression pattern of E-cadherin and $\beta$-catenin between the two types of endometrial carcinoma, we chose to compare similarly aggressive high-grade endometrioid adenocarcinomas with serous carcinomas.

\section{MATERIALS AND METHODS}

Seventeen cases of International Federation of Gynecology and Obstetrics (FIGO) Grade III endometrioid adenocarcinoma and 17 cases of serous carcinoma were selected from the archives of the New York Presbyterian Hospital. All cases were retrospectively reviewed by the authors, and the diagnoses were confirmed using accepted criteria (3). For demographic details, the reader is referred to previously published studies performed on the same cases $(25,26)$. One representative formalinfixed, paraffin-embedded tissue section from each case was submitted for immunohistochemistry. For antigen retrieval, pressure cooking or microwave pretreatment for 15 minutes was performed. Primary antibodies were obtained from Zymed Laboratories Inc. (South San Francisco, CA; E-cadherin, clone HECD-1; working dilution, 1:600) and Transduction Laboratories (Lexington, KY; $\beta$-catenin, clone 14 ; working dilution, 1:400). For visualization of the antigen, the 3,3'diaminobenzidine/peroxidase-based ChemMate kit (Ventana, Tucson, AZ) was used according to the manufacturer's instructions. All steps were carried out at room temperature.
Immunostains were evaluated independently by two authors (PWS and RAS) using a scoring protocol as described elsewhere (27). Briefly, a score ranging from 0 to 12 was determined as the product of staining intensity (on a scale from 0 to 3 ) and the percentage of positive cells (on a scale from 0 to 4 ). Scoring categories were defined with scores ranging from 0 (negative), 1 to 3 (weakly), 4 to 7 (moderately), and 8 to 12 (strongly positive). If the two observers' scores fell into the same category, their evaluation was considered concordant. If their scores fell into different categories, the third author's (LHE) evaluation was sought, and the disagreement settled by majority vote. For $\beta$-catenin, special consideration was given to the subcellular distribution of immunoreactivity (nuclear, membranous, or cytoplasmic).

For statistical analysis, the Student's $t$ test was used.

\section{RESULTS}

Nuclear expression of $\beta$-catenin was observed in 8 of 17 endometrioid adenocarcinomas but in none of 17 serous carcinomas $(P=.003)$. Conversely, moderate or strong E-cadherin expression was seen in 7 of 17 serous carcinomas but only in 1 of 17 endometrioid adenocarcinomas $(P=.02$; Figs. 2 and 3; Table 1). Of the 17 endometrioid adenocarcinomas, 7 showed strong nuclear $\beta$-catenin coupled with weak E-cadherin expression, 5 showed strong non-nuclear $\beta$-catenin coupled with weak E-cadherin expression, 1 showed strong nuclear $\beta$-catenin and strong E-cadherin expression, and 4 showed weak or no $\beta$-catenin coupled with weak E-cadherin expression. Thus, there was a tendency of strong $\beta$-catenin expression being associated with weak E-cadherin expression.

A comparable trend was not seen in serous carcinomas: of the 17 serous carcinomas, 5 showed strong non-nuclear $\beta$-catenin and strong E-cadherin expression, 5 had strong non-nuclear $\beta$-catenin and weak E-cadherin expression, 5 had both weak nonnuclear $\beta$-catenin and weak E-cadherin expression, and 2 had weak non-nuclear $\beta$-catenin with strong E-cadherin expression.

If present in serous carcinomas, $\beta$-catenin expression was non-nuclear. E-cadherin expression was restricted to the membranes in both histologic subtypes.

\section{DISCUSSION}

We undertook an immunohistochemical analysis of E-cadherin and $\beta$-catenin expression in serous carcinomas and poorly differentiated (FIGO Grade III) endometrioid adenocarcinomas. Using this 

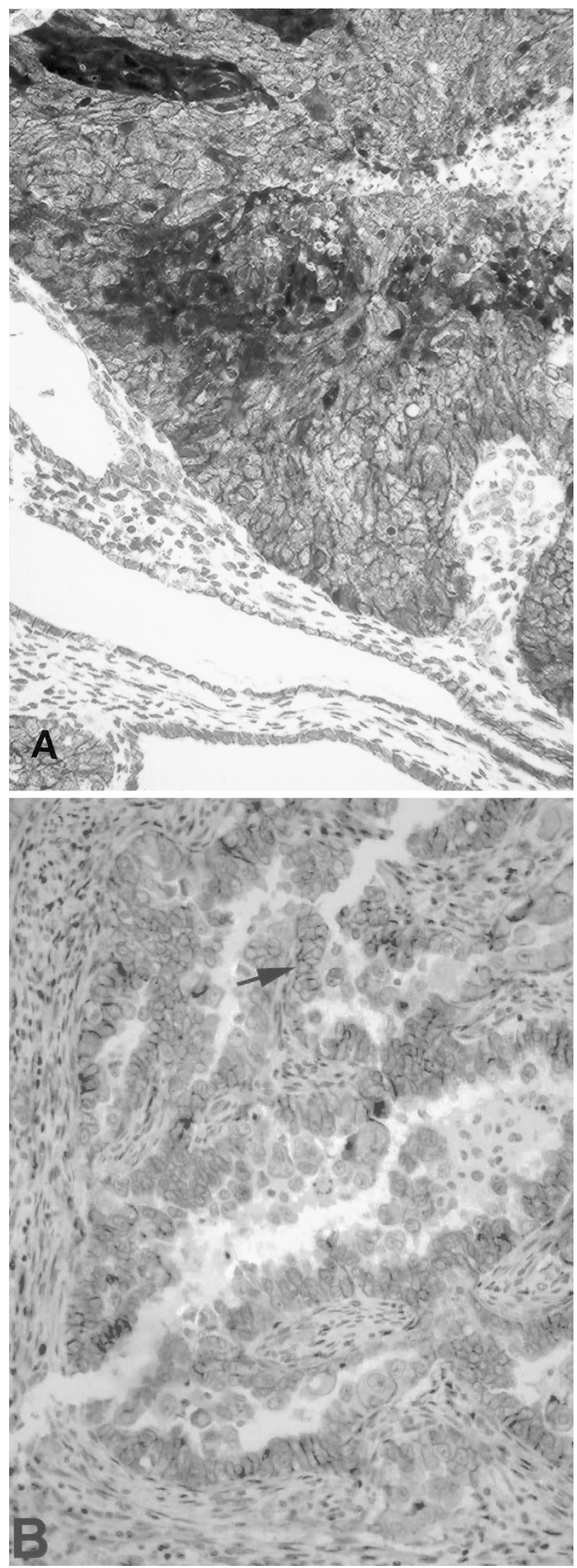

FIGURE 2. $\beta$-catenin immunostain. A, poorly differentiated (International Federation of Gynecology and Obstetrics Grade III) uterine endometrioid carcinoma: nuclear reactivity in numerous cells. B, uterine serous carcinoma: membranous reactivity is present (arrow), but nuclei are negative.

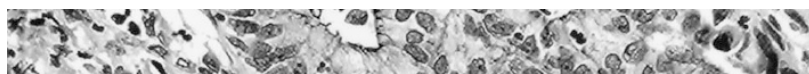

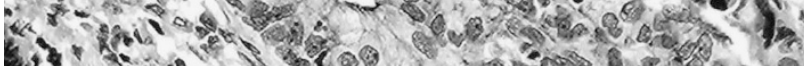

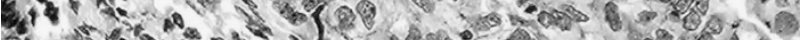

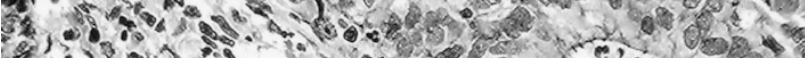
r.

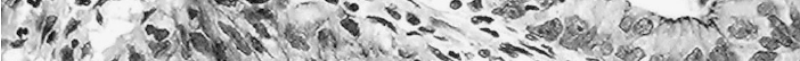

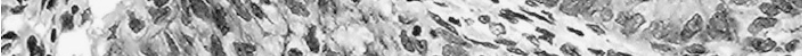

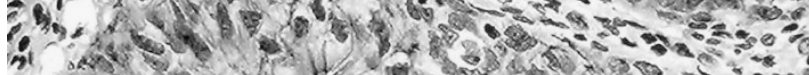

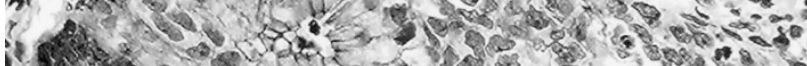

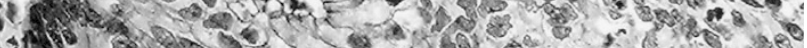

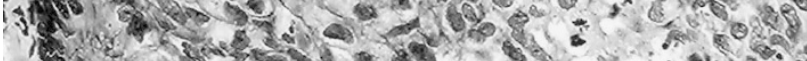

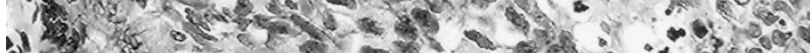

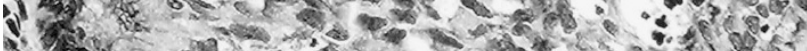

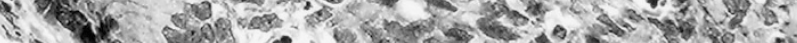

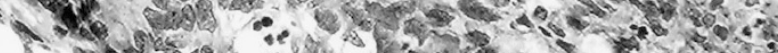
-

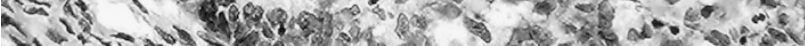

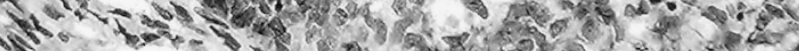

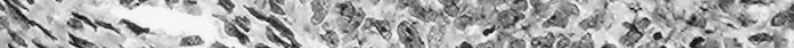

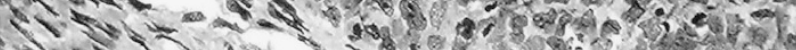

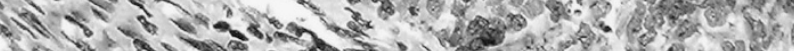

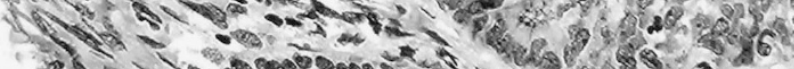

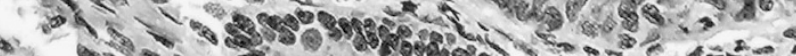

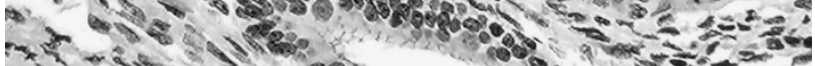

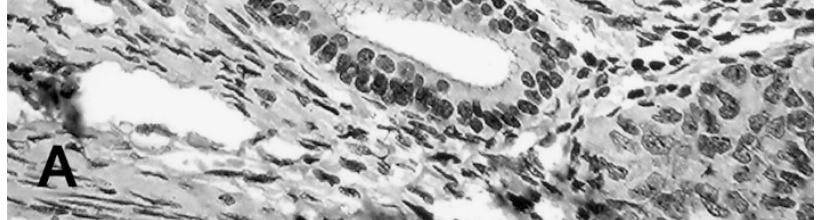
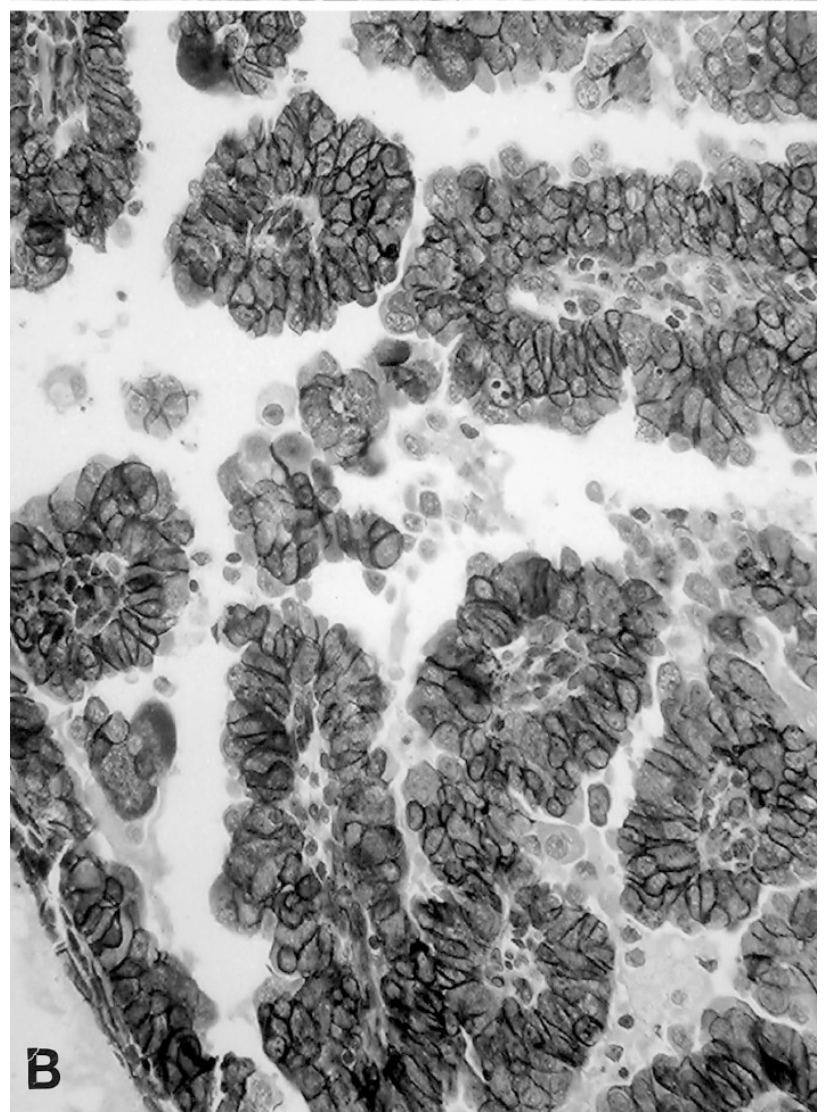

FIGURE 3. E-cadherin immunostain. A, poorly differentiated (International Federation of Gynecology and Obstetrics Grade III) uterine endometrioid carcinoma: focal weak expression on tumor cell membranes. Nuclei are negative. B, uterine serous carcinoma: strong membranous expression. Nuclei are negative. 
TABLE 1. Staining Patterns for $\beta$-Catenin and $e-$ Cadherin in Endometrioid Adenocarcinoma and Serous Carcinoma

\begin{tabular}{ccccccc}
\hline Staining Pattern & \multicolumn{2}{c}{ Endometrioid } & & \multicolumn{2}{c}{ Serous } & \\
\cline { 2 - 3 }$n$ & $n$ & $\%$ & & $n$ & $\%$ & \\
\hline $\begin{array}{c}\beta \text {-catenin nuclear } \\
\text { overexpression }\end{array}$ & $8 / 17$ & 47 & & $0 / 17$ & 0 & .003 \\
$\begin{array}{c}\text { E-cadherin } \\
\text { membranous } \\
\text { expression }\end{array}$ & $1 / 17$ & 6 & & $7 / 17$ & 41 & .02 \\
\hline
\end{tabular}

approach, we attempted to minimize a potential bias that might be introduced by comparing relatively indolent low-grade endometrioid adenocarcinomas with the generally highly aggressive serous carcinomas.

Nuclear expression of $\beta$-catenin was observed in a subset of endometrioid adenocarcinomas but not in serous carcinomas. Conversely, strong membranous E-cadherin expression was identified predominantly in serous carcinoma as compared with endometrioid adenocarcinoma. Most endometrioid adenocarcinomas showed strong $\beta$-catenin expression associated with weak E-cadherin expression. Thus, we found the expression patterns of E-cadherin and $\beta$-catenin to be strongly associated with the histologic subtype. This finding provides further support for the separation of endometrioid adenocarcinoma and serous carcinoma as different entities and for their distinct molecular genetic evolution.

Clinical, morphologic and molecular genetic evidence suggests that carcinoma of the endometrium is a heterogeneous group of diseases. Based on clinical data, two major types have been identified: Type I is estrogen related and tends to have a relatively favorable prognosis, whereas Type II is non-estrogen related and follows a more aggressive course (1). Morphologically, these two types can often be correlated with the endometrioid and serous variants of endometrial carcinoma, respectively (2). Recently, molecular genetic findings have supported the view that the two types of endometrial carcinoma emerge via different pathogenetic pathways. Notably, endometrioid adenocarcinomas show mutations in the PTEN tumor suppressor gene in up to $50 \%$ and a reported frequency of approximately $28 \%$ (5) of microsatellite instability in low-grade tumors, whereas p53 mutations tend to occur late in tumor development. In contrast, serous carcinomas harbor p53 mutations in approximately $90 \%$ early on but only rarely show PTEN mutations or microsatellite instability $(4,5)$.

Mutational analysis of CTNNB1 has revealed stabilizing $\beta$-catenin mutations in $10-20 \%$ of endometrioid carcinomas of the endometrium and of the ovary $(18-20,22,24)$. Similar figures have been described by Ikeda et al. (23), whereas MirabelliPrimdahl et al. (21) report a 33-50\% incidence of
CTNNB1 mutations in endometrial carcinomas; however, the histologic subtype of the analyzed cases was not clearly specified in these two studies. For unknown reasons, the mutations tend to occur in low-grade, low-stage tumors $(18,20,22,24)$ that lack lymph node metastases (28).

Immunohistochemically, nuclear accumulation of $\beta$-catenin has been reported in $\leq 38 \%$ of endometrioid tumors $(18,29)$. This suggests that abnormalities in other elements of the Wnt-signaling pathway may be involved in the pathogenesis of endometrioid carcinomas, the common result being an up-regulation of $\beta$-catenin. Saegusa and Okayasu (30) report an association of both $\beta$-catenin mutations and nuclear accumulation with squamous differentiation in G1 and G2 endometrioid endometrial and ovarian carcinomas.

The mutational status of CTNNB1 in serous carcinoma is unknown.

Cytoplasmic $\beta$-catenin is a key element in the Wnt signal transduction pathway, and its nuclear translocation has been linked to the induction of the $c$-myc proto-oncogene (12), cyclin D1 (13), and c-jun and fral (14), among others. Interestingly, cyclin D1 has been shown to be expressed in $48 \%$ of FIGO Grade III uterine endometrioid carcinomas, as opposed to only in $15 \%$ of uterine serous carcinomas (26). Thus, the similar percentage of cases positive for both $\beta$-catenin and cyclin D1 in high-grade endometrioid adenocarcinomas may reflect the induction of cyclin D1 by $\beta$-catenin overexpression.

In nonproliferating cells, the cytosolic $\beta$-catenin pool is strictly regulated by a phylogenetically highly conserved mechanism involving a multiprotein complex including the adenomatous polyposis coli tumor suppressor protein (APC), axin, and glycogen synthase kinase $3-\beta$ (GSK3 $\beta$ ), leading to phosphorylation and subsequent ubiquitindependent degradation of $\beta$-catenin (reviewed in $6-11)$. E-cadherin may contribute to the elimination of $\beta$-catenin from the cytoplasm by recruiting it into the adherens junction complex, thereby preventing its translocation into the nucleus $(31,32$, 33). On the other hand, as part of the adherens junction complex, $\beta$-catenin links E-cadherin through $\alpha$-catenin to the cytoskeleton (Fig. 1). Thus, malfunction of $\beta$-catenin may lead to decreased cell-cell adhesion and facilitate metastatic spread of carcinoma cells (17).

Altered E-cadherin expression has been associated with decreased cell-cell adhesion, metastatic potential, tumor dedifferentiation, and deep myometrial invasion in endometrial and other carcinomas $(15,16)$. Concerning the endometrioid tumors, our findings coincide with previous observations that poorly differentiated endometrial carcinomas are associated with decreased E-cadherin expression (16). Unfortunately, Sakuragi et al. (16) did not 
specify the histological subtype of the cases analyzed in their study. As our results show, the distinction of different subtypes of endometrial carcinoma is important, because serous tumors display a divergent expression pattern for E-cadherin.

Because both E-cadherin and $\beta$-catenin are involved in cell-cell adhesion, their differential expression pattern may also reflect the different modes of tumor dissemination in endometrioid adenocarcinomas versus serous carcinomas, the latter having a predilection for intraperitoneal dissemination.

Our results suggest that E-cadherin and $\beta$-catenin immunostains could be used along with p53 stains (5, 25) to differentiate between these two types of malignancies in diagnostically difficult situations.

In summary, we found the expression patterns of $\beta$-catenin and E-cadherin in high-grade endometrial carcinomas to be associated with the histological subtype. These findings support the concept of divergent molecular genetic pathways in different types of endometrial carcinomas. The immunohistochemical staining pattern could be useful for differentiating tumor types for diagnostic purposes.

Acknowledgments: The authors thank Ms. Liang Ying for excellent technical assistance.

\section{REFERENCES}

1. Bokhman JV. Two pathogenetic types of endometrial carcinoma. Gynecol Oncol 1983;15:10-7.

2. Deligdisch L, Cohen CJ. Histologic correlates and virulence implications of endometrial carcinoma associated with adenomatous hyperplasia. Cancer 1985;56:1452-5.

3. Kurman RJ, Zaino RJ, Norris HJ. Endometrial carcinoma. In: Kurman RJ, editor. Blaustein's pathology of the female genital tract. 5th ed. New York: Springer Verlag; 2002.

4. Hedrick L. Endometrial cancer. In: Vogelstein B, Kinzler K, editors. The genetic basis of human cancer. New York: McGraw-Hill; 1998.

5. Lax SF, Kendall B, Tashiro H, Slebos RJ, Hedrick L. The frequency of $p 53, K$-ras mutations, and microsatellite instability differs in uterine endometrioid and serous carcinoma: evidence of distinct molecular genetic pathways. Cancer 2000;88:814-24.

6. Morin PJ. $\beta$-catenin signaling and cancer. Bioessays 1999;21: 1021-30.

7. Behrens J. Cadherins and catenins: role in signal transduction and tumor progression. Cancer Metastasis Rev 1999;8: 15-30.

8. Beavon IRG. The E-cadherin-catenin complex in tumour metastasis: structure, function and regulation. Eur J Cancer 2000;36:1607-20.

9. Polakis P. Wnt signaling and cancer. Genes Dev 2000;14: 1837-51.

10. Peifer M, Polakis P. Wnt signaling in oncogenesis and embryogenesis—a look outside the nucleus. Science 2000;287: 1606-9.

11. Wijnhoven BPL, Dinjens WNM, Pignatelli M. E-cadherincatenin cell-cell adhesion complex and human cancer. $\mathrm{Br}$ J Surg 2000;87:992-1005.

12. He T-C, Sparks AB, Rago C, Hermeking H, Zawel L, Da Cost LT, et al. Identification of c-MYC as a target of the APC pathway. Science 1998;281:1509-12.
13. Tetsu O, McCormick F. $\beta$-Catenin regulates expression of cyclin D1 in colon carcinoma cells. Nature 1999;398: 422-6.

14. Mann B, Gelos M, Siedow A, Hanski ML, Gratchev A, Ilyas M, et al. Target genes of $\beta$-catenin $\mathrm{T}$ cell-factor lymphoidenhancer-factor signaling in human colorectal carcinomas. Proc Natl Acad Sci U S A 1999;96:1603-8.

15. Fujimoto J, Ichigo S, Hirose R, Sakaguchi H, Tamaya T. Suppression of E-cadherin and alpha- and beta-catenin mRNA expression in the metastatic lesions of gynecological cancers. Eur J Gynaecol Oncol 1997;18:484-7.

16. Sakuragi N, Nishiya M, Ikeda K, Ohkouch T, Furth EE, Hareyama $\mathrm{H}$, et al. Decreased E-cadherin expression in endometrial carcinoma is associated with tumor dedifferentiation and deep myometrial invasion. Gynecol Oncol 1994; 53:183-9.

17. Oyama T, Kanai Y, Ochiai A, Akimoto S, Oda T, Yanagihara K, et al. A truncated beta-catenin disrupts the interaction between E-cadherin and alpha-catenin: A cause of loss of intercellular adhesiveness in human cancer cell lines. Cancer Res 1994;54:6282-7.

18. Fukuchi T, Skamoto M, Tsuda H, Maruyama K, Nozawa S, Hirohashi S. $\beta$-catenin mutation in carcinoma of the uterine endometrium. Cancer Res 1998;58:3526-8.

19. Palacios J, Gamallo C. Mutations in the $\beta$-catenin gene (CTNNB1) in endometrioid ovarian carcinomas. Cancer Res 1998;58:1344-7.

20. Kobayashi K, Sagae S, Nishioka Y, Tokino T, Kudo R. Mutations of the $\beta$-catenin gene in endometrial carcinomas. Jpn J Cancer Res 1999;90:55-9.

21. Mirabelli-Primdahl L, Gryfe R, Kim H, Millar A, Luceri C, Dale D, et al. $\beta$-catenin mutations are specific for colorectal carcinomas with microsatellite instability but occur in endometrial carcinomas irrespective of mutator pathway. Cancer Res 1999;59:3346-51.

22. Wright K, Wilson P, Morland S, Campbell I, Walsh M, Hurst $\mathrm{T}$, et al. $\beta$-catenin mutation and expression analysis in ovarian cancer: exon 3 mutations and nuclear translocation in $16 \%$ of endometrioid tumours. Int $\mathrm{J}$ Cancer 1999;82:625-9.

23. Ikeda T, Yoshinaga K, Semba S, Kondo E, Ohmori H, Horii A. Mutational analysis of the CTNNB1 beta-catenin gene in human endometrial cancer: frequent mutations at codon 34 that cause nuclear accumulation. Oncol Rep 2000;7:323-6.

24. Schlosshauer PW, Pirog EC, Levine RL, Hedrick Ellenson L. Mutational analysis of the CTNNB1 and APC genes in uterine endometrioid carcinoma. Mod Pathol 2000;13:1066-71.

25. Soslow RA, Shen PUF, Chung MH, Isacson C. Distinctive p53 and mdm2 immunohistochemical expression profiles suggest different pathogenetic pathways in poorly differentiated endometrial carcinoma. Int J Gynecol Pathol 1998;17:12934 .

26. Soslow RA, Shen PUF, Chung M, Isacson C, Baergen RN. Cyclin D1 expression in high grade uterine carcinoma correlates with histologic subtype and estrogen receptor expression. Int J Gynecol Pathol 2000;19:329-34.

27. Remmele W, Schicketanz K-H. Immunohistochemical determination of estrogen and progesterone receptor content in human breast cancer. Computer-assisted image analysis (QIC score) vs. subjective grading (IRS). Pathol Res Pract 1993;189:862-6.

28. Saegusa M, Hashimura M, Yoshida T, Okayasu I. $\beta$-catenin mutations and aberrant nuclear expression during endometrial tumorigenesis. Br J Cancer 2001;84:209-17.

29. Nei H, Saito T, Yamasaki H, Mizumoto H, Ito E, Kudo R. Nuclear localization of beta-catenin in normal and carcinogenic endometrium. Mol Carcinog 1999;25:207-18. 
30. Saegusa M, Okayasu I. Frequent nuclear $\beta$-catenin accumulation and associated mutations in endometrioid-type endometrial and ovarian carcinomas with squamous differentiation. J Pathol 2001;194:59-67.

31. Hulsken J, Birchmeier W, Behrens J. E-cadherin and APC compete for the interaction with beta-catenin and the cytoskeleton. J Cell Biol 1994;127:2061-9.
32. Fagotto F, Funayama N, Gluck U, Gumbiner BM. Binding to cadherins antagonizes the signaling activity of beta-catenin during axis formation in Xenopus. J Cell Biol 1996;132:1105-14.

33. Yang S-Z, Kohno N, Yokoyama A, Kondo K, Hamada H, Hiwada K. Decreased E-cadherin augments $\beta$-catenin nuclear localization: studies in breast cancer cell lines. Int J Oncol 2001;18:541-8.

\section{Book Review}

\section{Rowan RM, van Assendelft OW, Preston FE, editors: Ad- vanced Laboratory Methods in Haematology, 452 pp, London, Arnold, 2002 (\$98.50).}

A sea change has occurred in hematology over the past 40 years. Clinical laboratories have offered groups of hematology tests as a "complete blood count" (the CBC). While "can do" automation has partly defined the content of chemistry panels, multichannel hematology analyzers are committed to the $\mathrm{CBC}$, in previous years based on manual methods but now requiring pneumatic systems for cell transport. Standardization and adherence to strict control procedures is required. These considerations are focal points for the recent text Advanced Laboratory Methods in Haematology. In just over 400 pages, this book provides a comprehensive review of hematology testing that has evolved over the past $40-50$ years to the current age of automated multichannel devices.

In the preface, editor/authors RM Rowan, OW van Assendelft, and FE Preston summarize the functions of the International Council (formerly committee) for Standardization in Haematology (ICSH). While the text under review is said to be "intended for haematologists and technical staff in haematology laboratories" (and for students, teachers, and administrators), the book will be of value to additional professionals. In a sense, the title Advanced Laboratory Methods in Haematology is a misnomer in that the text has broader application. Clinical relevance is woven liberally into the text.

An introduction follows the preface. Here, the evolution of a number of prestigious societies and committees is followed to the emergence of the ICSH in 1966. The text under review is the latest and most comprehensive publication in a series relating to the ICSH. The authors (there are 22 contributors) are established authorities in hematology; some are members (or founders) of the ICSH.

The text consists of 18 chapters grouped into six parts titled: The Blood Count, Haemoglobinometry, Haemoglobin A2F and the Abnormal Haemoglobins, Erythrocyte Sedimentation, Haematopoietic Factors, and Coagulation Testing. Sophisticated but highly readable and thorough discussions, inclusive of some test methods, form the content of individual chapters. Specimen handling, standardization, and quality assurance are especially emphasized.

Six chapters form Part 1, The "Blood Count." The first chapter is devoted entirely to quality assurance. The College of American Pathologists "Q-probe" studies (e.g., turnaround time) are reviewed. The subsequent chapters deal with "The Blood Cell Count," "The Differential Cell Count," "Instrumental Flagging and Blood Film Review," "Reticulocyte Counting” (including clinical applications), and "Leukocyte Immunophenotyping." Basic through advanced material is presented. Discussions include such topics as "data overload," relation of sequential data to clinical situations, application of monoclonal antibodies to five-cell differential counts, and bone marrow study by cell analyzers. Abundant appropriate tables, charts, and diagrams include representation of blood cell differentiation; a table of reticulocyte flow cytometer procedures; cells and particles (total of 22) that may interfere with the reticulocyte count; reference ranges for reticulocyte parameters; a table of $\mathrm{CD}$ (cluster of differentiation) designates (four pages inclusive of CD 1 a,b,c, through CD 166); an excellent detailed schematic diagram of flow cytometry, immunophenotyping, and classification of leukemias; and many others.

The two chapters of Part 2 deal with hemoglobinometry. The first reviews technical aspects; the second is clinically oriented. Standardization is emphasized. Included are sections on spectrophotometry, role of the ICSH, haemiglobincyanide standards, and standard solutions, their preparation, and stability.

Part 3 (with a single chapter) covers the important areas of hemoglobinopathy. Various electrophoretic methods (including isoelectric focusing) are presented. Procedures are detailed including methods for Hgbs F, A, the sickling, and the unstable hemoglobins. Appropriate diagrams and charts are included. Separate sections discuss the thalassemias and neonatal screening. Part 4 (also a single chapter) reviews erythrocyte sedimentation and includes discussions of theoretic and clinical aspects, as well as early and current instrumentation. There is a comment that the rapid, automated zeta sedimentation rate device is "no longer marketed." The zeta fuge, however, is now produced in China and has been used in a study of zeta reference values published in the Chinese literature.

The two chapters of Part 5, "Haematopoetic Factors," cover ferritin, serum B12, serum/red cell folate, and include discussions of carcino-fetal ferritins, serum homocysteine, and methylmalonic acid. The last part (6), concerns "Coagulation Testing," and has six chapters. Monitoring of heparin and oral anticoagulant (warfarin based) therapy is thoroughly explored. Critical questions about the near universally accepted International Normalized Ratio (INR) are considered. A section is devoted to point-of-care testing. Separate chapters present the subjects of reference ranges, lupus anticoagulant testing, and familial thrombophilia.

Each chapter of this book is extensively referenced (alphabetically by author). A detailed index forms the final 15 pages of this outstanding text. Advanced Laboratory Methods in Haematology is an important, well-written, and informative text of hematology and coagulation. Its sophisticated clinical laboratory perspective does not detract from practical and useful clinical application. This book is highly recommended not only to clinical and laboratory practitioners of hematology but also to medical professionals, students, teachers, and administrators extending beyond the target audience.

\author{
Wayne R. DeMott \\ University of Kansas Medical Center \\ Kansas City, Kansas
}

